\title{
La imagen social de la enfermería: una profesión a conocer
}

\section{The public image of nursing: a profession to learn about}

\author{
B. Errasti-Ibarrondo ${ }^{1}$, M. Arantzamendi-Solabarrieta ${ }^{1}$, N. Canga-Armayor ${ }^{2}$
}

\section{RESUMEN}

La enfermería es una profesión que ha sufrido del estereotipo público durante mucho tiempo, estando sujeta a ser vista como una profesión predominantemente femenina, bajo el mandato médico y sin un campo competencial propio. Es innegable que en la última década se están sucediendo cambios trascendentales para la disciplina enfermera. En consecuencia, a través de esta revisión, con metodología sistemática, se pretende analizar la imagen social de la enfermería para poder vislumbrar si la población está percibiendo los cambios que se están sucediendo y si tiene una imagen cercana de lo que es o pretende ser. Tras realizar un análisis temático de las publicaciones seleccionadas han emergido ocho categorías, que pueden ayudar a explicar la visión que tiene la sociedad sobre la enfermería. Parece que predomina una ignorancia del público sobre la esencia y el foco de la disciplina, que es el cuidado de la persona, familia o comunidad. Asimismo, es una profesión poco visible ya que la sociedad todavía no la reconoce plenamente con un campo competencial propio. Con respecto a la autonomía e independencia de las enfermeras, se constata que hay una tendencia a verla como una profesión inferior a la medicina. Se percibe como una profesión estrechamente relacionada con "tareas" y se confía en ella para las actividades que le han sido asociadas tradicionalmente. No obstante, se va percibiendo como una profesión menos relacionada con la feminidad y más preparada que antaño.

Palabras clave. Enfermería. Imagen social. Imagen pública. Imagen de la enfermería. Estereotipo.

\begin{abstract}
Nursing is a profession that has suffered from public stereotyping for a long time. It has been viewed historically as a female profession, under the orders of doctors and without its own field of competence. Major changes have occurred in the discipline over the last decade that directly affect the profession. As a result, this review of the literature has tried to identify the image of nursing held by the population, the prevailing stereotypes related to it, and what the public considers that nurses do.

Eight categories emerged from the thematic analysis that can help in explaining the view that society has of nursing and the evolution it has undergone. It seems that the public is predominantly ignorant of the essence and focus of the discipline, which is none other than care of the person, family or community. It is also a little known profession as society does not fully acknowledge that nurses have their own field of competence, autonomy and independence. There is still a tendency to consider nursing as a profession inferior to medicine, closely related to "tasks", and there is trust in its carrying out those activities that have been traditionally associated with it. However, it is coming to be perceived as a profession that is less related to femininity and that involves greater preparation than in the past.
\end{abstract}

Key words. Nursing. Public image. Nursing Stereotypes. Social image.
1. Departamento de Enfermería de la Persona Adulta. Facultad de Enfermería. Universidad de Navarra. Pamplona.

2. Departamento de Enfermería Comunitaria y Materno Infantil. Facultad de Enfermería. Universidad de Navarra. Pamplona.

\section{Correspondencia:}

Begoña Errasti-Ibarrondo

Departamento de Enfermería de la Persona Adulta Facultad de Enfermería. Universidad de Navarra Irunlarrea, 1. 31008 Pamplona (Navarra) España E-mail: meibarrondo@unav.es

Recepción: 5 de diciembre de 2011

Aceptación provisional: 16 de febrero de 2012

Aceptación definitiva: 12 de marzo de 2012 


\section{INTRODUCCIÓN}

Si en alguna cosa existe un amplio consenso en relación a la enfermería es su dificultad para hacerse claramente visible e identificable ${ }^{1}$. Collière afirmó que a menudo el cuidado es "invisible ${ }^{2}$ y Kèrouac y $\mathrm{col}^{2}$ describieron entre otras como acciones invisibles, el hecho de cuidar o preocuparse de alguien. Esto puede suponer que el quehacer enfermero resulte difícil de ver cuando está, pero que entrañe un gran vacío en su ausencia.

La enfermería ha sufrido del estereotipo público durante mucho tiempo ${ }^{3}$. En la literatura han sido descritas numerosas veces los clichés a los que se le ha vinculado a lo largo de la historia y los principales factores que han contribuido a ello ${ }^{4,5}$. Algunos estudios realizados sobre la imagen pública de las enfermeras tienden a mostrar que a pesar del tiempo, el público continúa percibiéndolas a través de estereotipos y que éstos, han mejorado poco a lo largo del tiempo ${ }^{3,4}$. Una de las causas atribuidas a la consolidación de una imagen enfermera que poco tiene que ver con la realidad actual es la estructura jerárquica entre el médico y la enfermera, que emanan de sus respectivos orígenes educacionales ${ }^{3,6}$. Hay que tener en cuenta que la enfermería ha sido una profesión ejercida mayoritariamente por mujeres y que se ha desarrollado tradicionalmente junto a profesionales médicos ${ }^{7}$, bajo su supervisión y siendo considerados como un colectivo de gran prestigio $^{8}$. De modo que "la contribución de la profesión enfermera ha sido percibida como la prolongación del servicio que los médicos daban a la población $»^{2}$. Además, no debemos olvidar que la enfermería ha sido estrechamente vinculada a un estereotipo sexual ${ }^{3,6}$, y que en los medios de comunicación se ha reforzado una imagen tradicional sobre la enfermería ${ }^{3,9}$. Estos son sólo algunos de los condicionantes que han podido llevar a la población a tener una idea equivocada o incompleta de lo que es el quehacer enfermero, no contemplando el propio campo de responsabilidad de la enfermería.

Es indiscutible que la imagen es un valor que prima en la sociedad actual. Una imagen negativa de la enfermería puede tener numerosas consecuencias ${ }^{10}$, como que la población no recurra a ella y no se beneficie de sus servicios. Asimismo, puede tener un impacto en la cantidad y calidad de personas que la eligen como profesión ${ }^{3,10}$, o que afecte a la toma de decisiones de los políticos, que definen el alcance y la financiación de los servicios ${ }^{10}$. Por lo tanto, el modo en que los demás perciben a la Enfermería puede ser decisivo para establecer su participación en el proceso de construcción de la política pública ${ }^{11}$.

La enfermería ha evolucionado en el contexto de los acontecimientos históricos y movimientos sociales sucedidos durante estos últimos siglos ${ }^{2}$. En esta última década se han dado cambios con repercusiones trascendentales para la enfermería, impulsados principalmente por la aprobación de la Ley de Ordenación de las Profesiones Sanitarias y por la reestructuración de los estudios universitarios, con la implantación del grado y el postgrado (máster y doctorado). De modo que estamos en un periodo de ampliación de funciones, crecimiento y enriquecimiento de la formación académica e investigadora, a través de las cuales vamos a ir desarrollando nuestro propio cuerpo de conocimientos y avanzando como disciplina, aguardándonos un futuro con grandes expectativas y responsabilidad con la sociedad.

En principio, toda persona tiene una idea de lo que es la enfermería y cuál es su papel en la sociedad. Sin embargo, de las interpretaciones que habitualmente hace la población puede deducirse que es una profesión profundamente desconocida, de la que aún persisten ciertos estereotipos que nada tienen que ver con lo que hoy es, y con la proyección que tiene para el futuro.

Considerando la situación académica actual de la Enfermería, como un punto de inflexión histórico para la disciplina, con esta revisión se pretende analizar la imagen social para poder vislumbrar si la población está percibiendo los cambios que se están sucediendo y si tiene una imagen cercana de lo que es o pretende ser. 


\section{MATERIAL Y MÉTODOS}

Se llevó a cabo una revisión de la literatura con metodología sistemática entre diciembre de 2010 y abril de 2011 siguiendo la secuencia que se describe a continuación:

1. Establecimiento de los criterios de inclusión y de exclusión (Tabla 1) y los límites (publicaciones en inglés o español).

2. Diseñar la estrategia de búsqueda identificando los aspectos principales a estudiar (imagen, enfermería y sociedad), así como los términos a incluir en cada uno de ellos (Fig. 1).

3. Realización de búsquedas en bases de datos específicas de enfermería: Cinahl y Cuiden.
4. Realización de búsquedas en bases de datos de referencia (Pubmed y PsycInfo) y otras (Fundación Dialnet).

5. Selección de los estudios tras la aplicación de los criterios de inclusión y de exclusión.

6. Revisión de las listas de referencias de los artículos seleccionados.

7. Evaluación de la calidad de los estudios seleccionados en base a «Scottish Intercollegiate Guidelines Network. A guideline developer's handbook» ${ }^{12}$.

8. Análisis temático de las publicaciones seleccionadas.

Tabla 1. Criterios de inclusión y de exclusión

\begin{tabular}{|c|c|}
\hline Criterios de inclusión & Criterios de exclusión \\
\hline $\begin{array}{l}\text { Estudios que tengan como objetivo principal identifi- } \\
\text { car y/o describir la imagen o los posibles estereotipos } \\
\text { predominantes en la sociedad sobre la enfermería }\end{array}$ & $\begin{array}{l}\text { Estudios que identifiquen, describan y/o analicen la } \\
\text { imagen o estereotipos enfermeros en los medios de } \\
\text { comunicación/ Mass Media (T.V, prensa, cine, inter- } \\
\text { net, etc.) }\end{array}$ \\
\hline $\begin{array}{l}\text { Estudios que tengan como objetivo principal o secun- } \\
\text { dario describir qué es lo que hacen las enfermeras } \\
\text { según la sociedad/población }\end{array}$ & $\begin{array}{l}\text { Estudios realizados con profesionales sanitarios: } \\
\text { Enfermeras (estudiantes/ titulares) } \\
\text { Otros miembros del equipo de cuidados }\end{array}$ \\
\hline Estudios realizados con adultos mayores de 18 años* & Estudios realizados sobre la literatura profesional \\
\hline Estudios realizados con ciudadanos & $\begin{array}{l}\text { Estudios realizados sobre la imagen oficial (documen- } \\
\text { tos institucionales) }\end{array}$ \\
\hline $\begin{array}{l}\text { Estudios realizados con clientes/personas que hayan } \\
\text { tenido experiencias o no con la asistencia sanitaria }\end{array}$ & $\begin{array}{l}\text { Estudios realizados con estudiantes universitarios o } \\
\text { niños }\end{array}$ \\
\hline \multirow[t]{3}{*}{ Tesis doctorales y disertaciones } & $\begin{array}{l}\text { Literatura gris (folletos, editoriales, noticias...) y pu- } \\
\text { blicaciones en congresos }\end{array}$ \\
\hline & $\begin{array}{l}\text { Estudios que se centren en una única especialidad } \\
\text { enfermera }\end{array}$ \\
\hline & Texto completo no disponible \\
\hline
\end{tabular}

*Se incluyeron dos estudios que incluían algunos jóvenes menores de 18 años por considerar no significativo el número de estos sujetos en relación al resto de la población estudiada. 


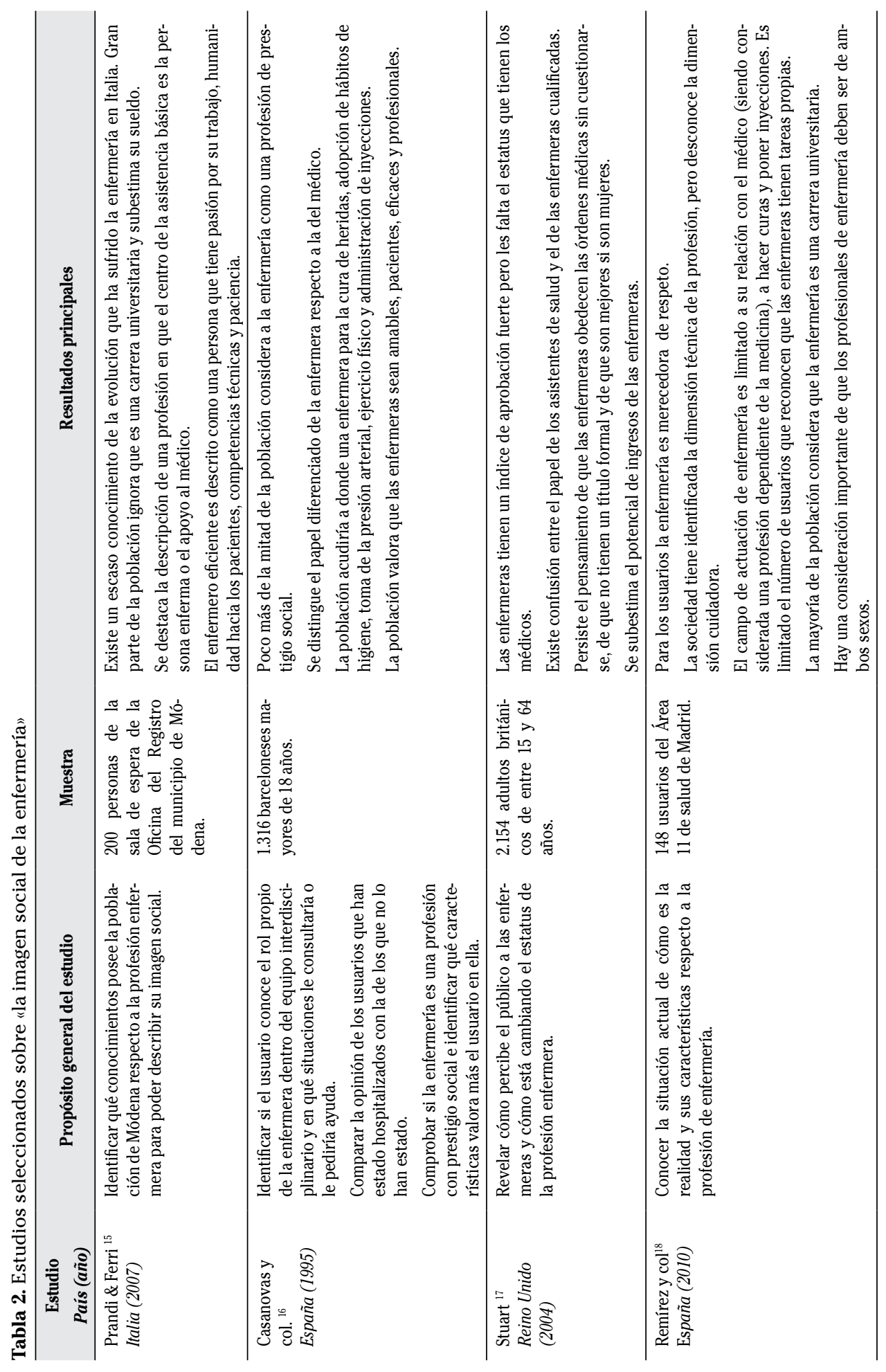




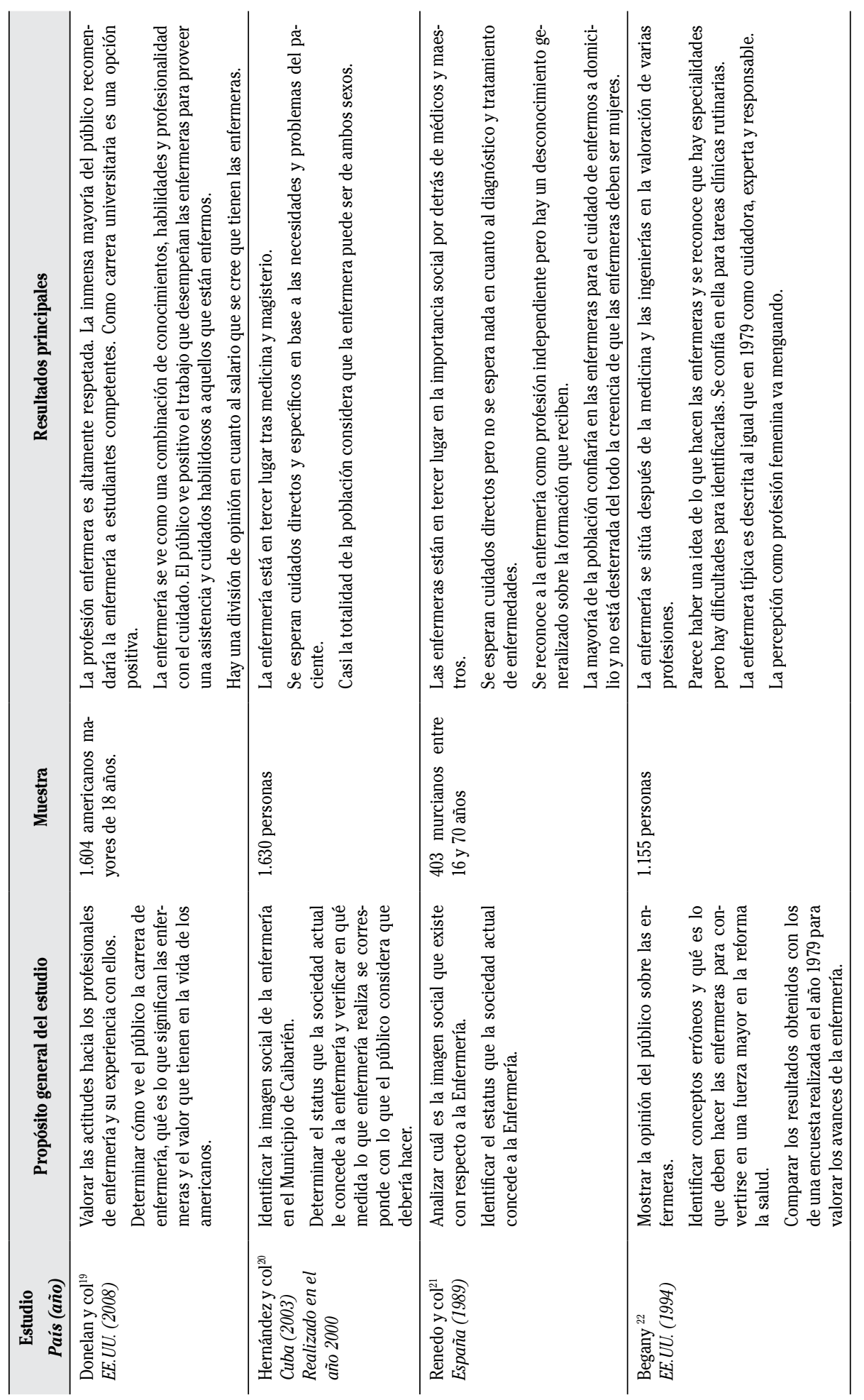




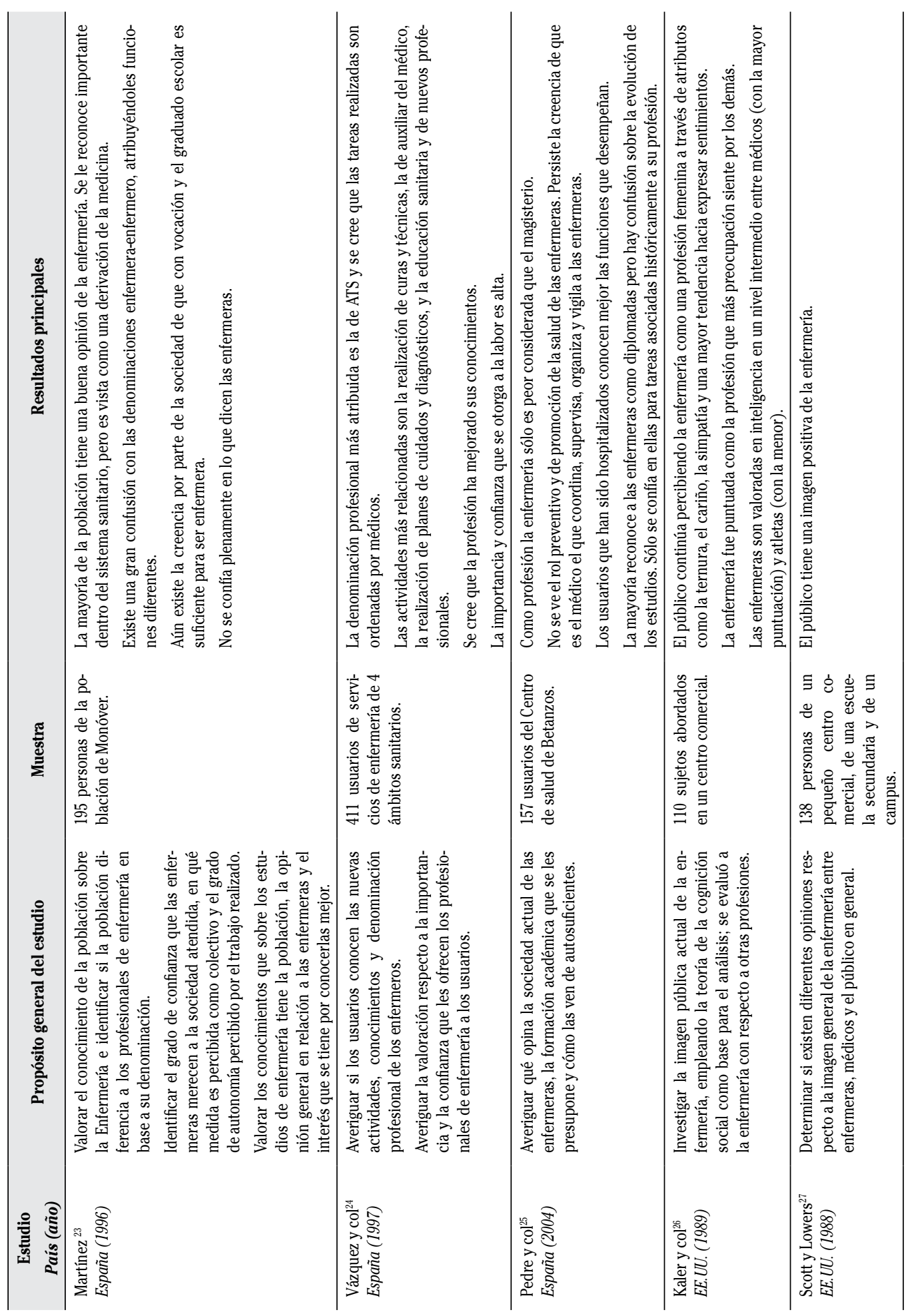




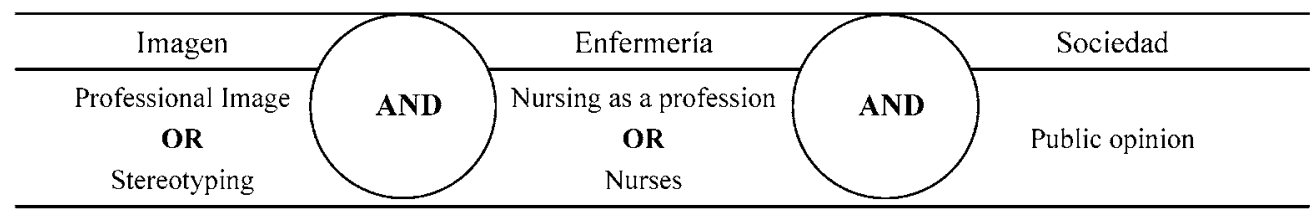

Figura 1. Aspectos principales y términos generales de la revisión.

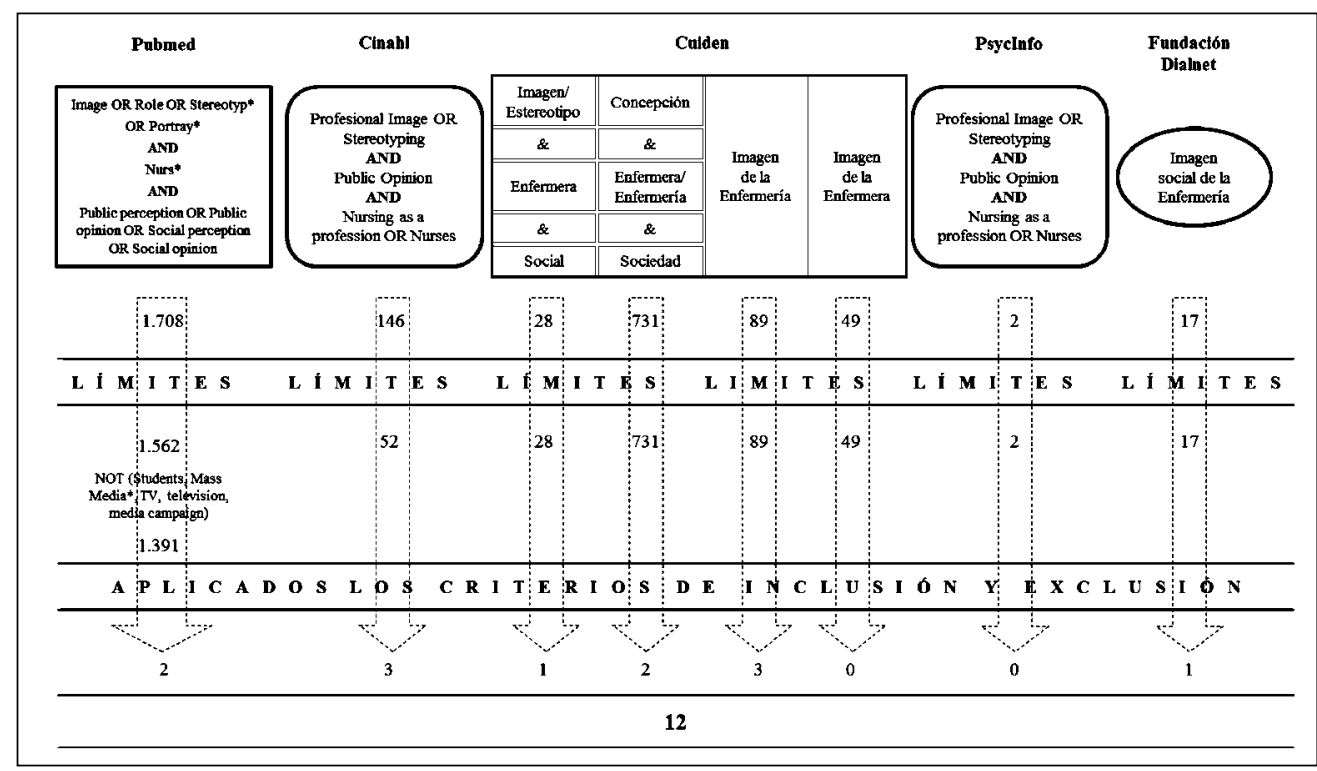

Figura 2 Proceso de las búsquedas en las distintas bases de datos y resultados obtenidos.

\section{RESULTADOS}

En la búsqueda bibliográfica se identificaron doce artículos y tras la revisión de las listas de referencia se añadió uno, obteniendo un total de trece artículos. Las publicaciones seleccionadas proceden de cinco países y abarcan el periodo comprendido entre 1988 y 2010 . Todos los estudios tienen un diseño cuantitativo descriptivo de corte transversal y emplean un cuestionario como instrumento de medida. Asimismo, todos han sido valorados con un nivel de evidencia $3^{\text {a }}$. La tabla 2 muestra los artículos seleccionados y la tabla 3 refleja los principales aspectos metodológicos de los estudios.

Puesto que el propósito de esta revisión era analizar la imagen o concepto que tiene la sociedad sobre la enfermería, a pesar de valorar el nivel de evidencia, el análisis de los estudios se centró en el análisis temático. 


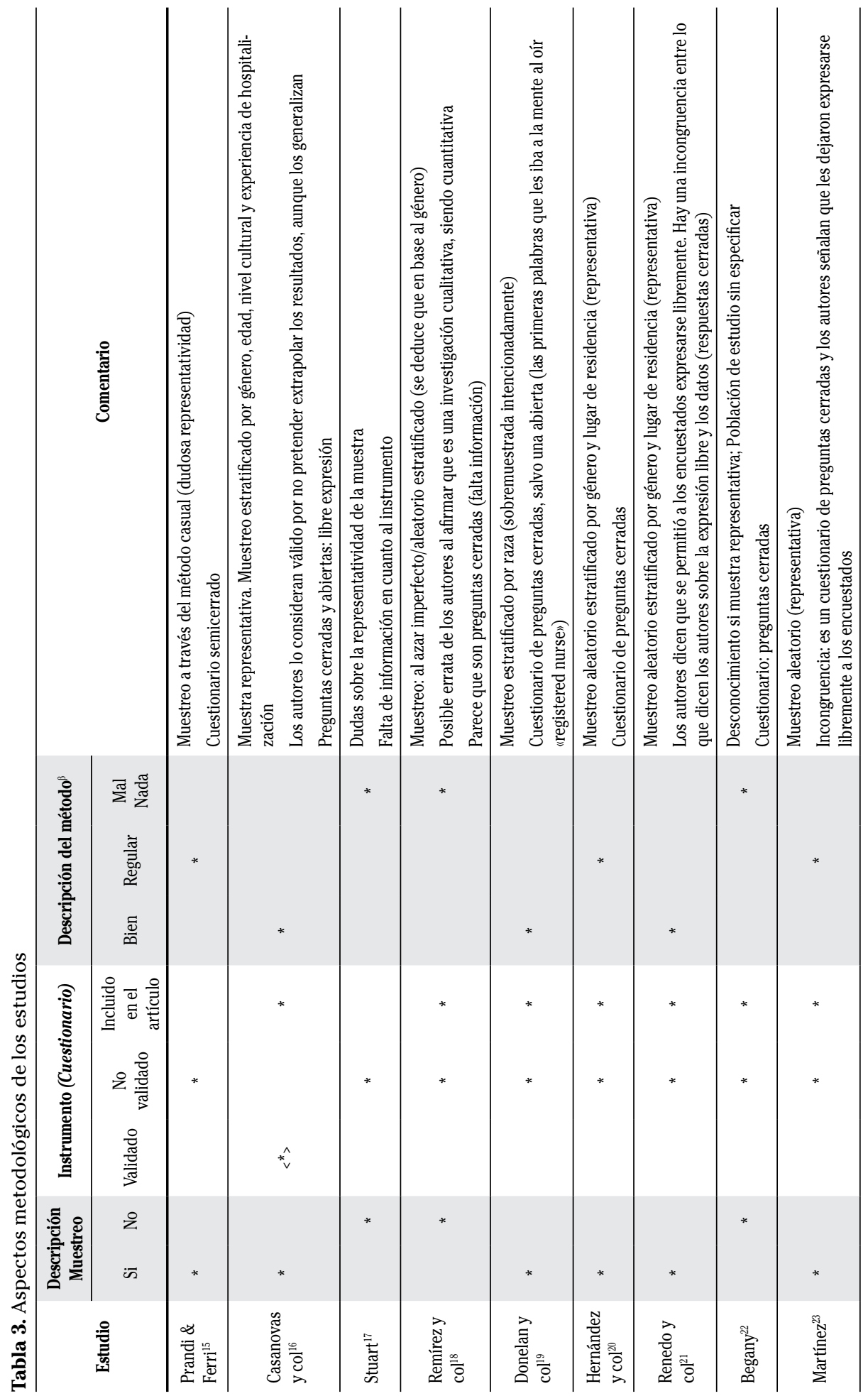




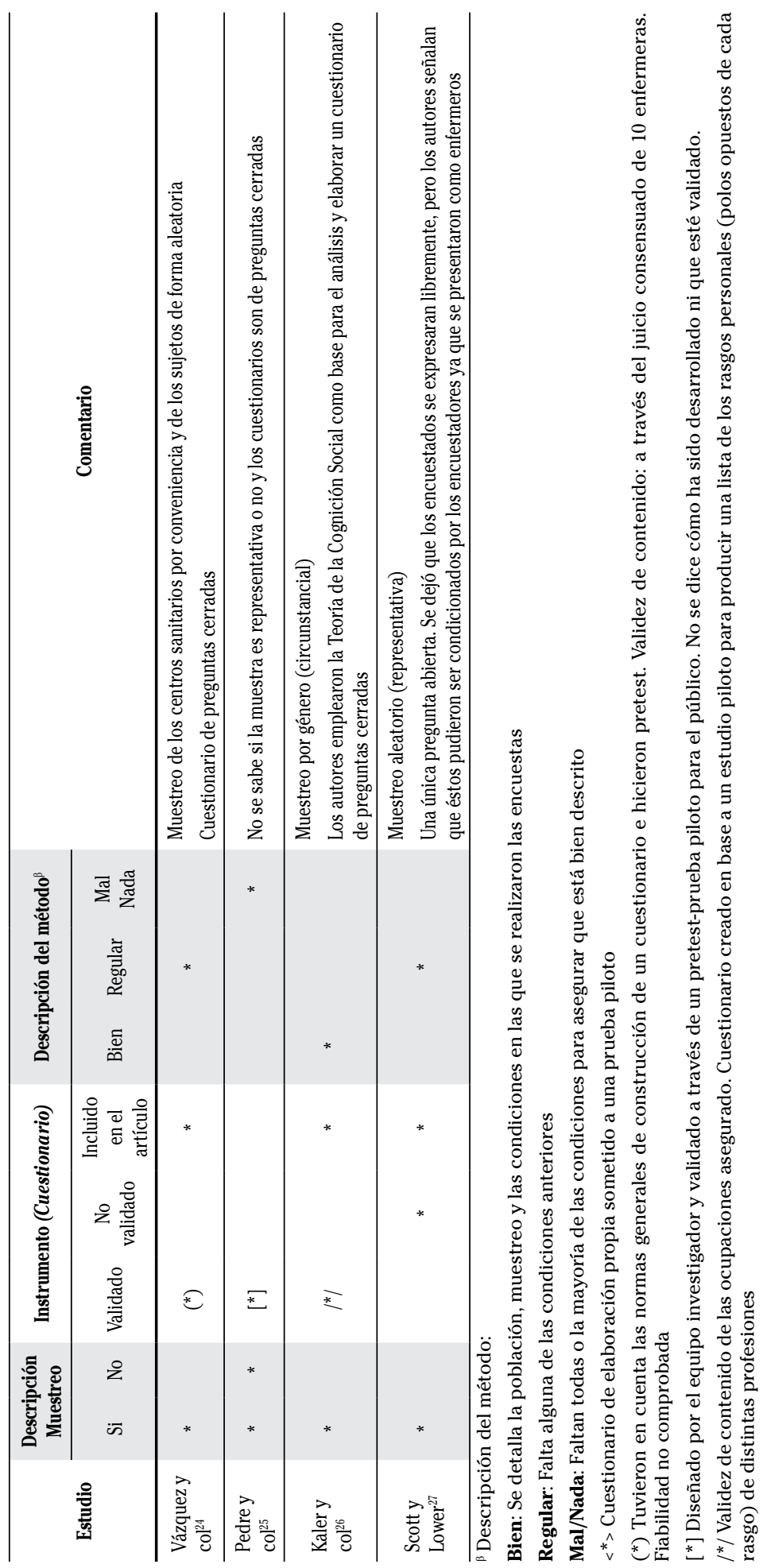




\section{ANÁLISIS TEMÁTICO: CATEGORÍAS EMERGENTES}

Se realizó un análisis temático de los estudios para tratar de resumir sus resultados en líneas temáticas ${ }^{13}$ siguiendo el siguiente esquema: lectura en profundidad de los artículos seleccionados; análisis de la información, identificación de cuestiones que respondieran al objetivo de la búsqueda y de conceptos relevantes; identificación de temas importantes o recurrentes de las publicaciones; clasificación de los artículos en base a las líneas temáticas emergidas; resumen de los resultados de los artículos en dichas líneas temáticas.

Consecuentemente, emergieron ocho categorías que ayudan a explicar la visión que tiene la sociedad sobre la enfermería: estatus y/o prestigio social, funciones y/o actividades, independencia, formación, atributos de las enfermeras, salario, confianza en el profesional de enfermería, género vinculado a la enfermería. El conjunto de estos ocho temas principales pueden constituir lo que Chombard de Lauwe (1963) definió como imagen ${ }^{14}$.

\section{Estatus y/o prestigio social}

Se aborda la posición que otorga la población a la enfermería como profesión y/o como carrera universitaria con respecto a otras.

Aunque la consideración social de la enfermería sea escasa ${ }^{15-17}$, como carrera universitaria es vista como una opción positiva $^{15,18,19}$, constatándose que muchos padres aconsejan a sus hijos estudiar enfermería $^{15,18}$. Sin embargo, parece que la profesión carece del estatus que tienen los médicos ${ }^{17}$, lo que es corroborado por otros estudios, en los que se concluye que la enfermería ocupa el tercer lugar en la valoración de varias profesiones situándose por detrás de la medicina $^{20-22}$. Pese a ello, y no siendo un resultado contradictorio, se reconoce importante su rol dentro del sistema sanitario ${ }^{23}$.

\section{Funciones y/o actividades}

Engloba aspectos como qué espera la sociedad de la enfermería y las "tareas" y/o funciones que cree que realiza o desempeña. Se aborda también la identificación de los profesionales según su denominación.

El trabajo enfermero es descrito mediante muchas actividades ${ }^{15}$, siendo las más relacionadas con la profesión el poner inyecciones ${ }^{15,16,18,20-22}$, el curar heridas $^{16,18,20,21,24}$, o el ayudar al médico ${ }^{15,18,20,21,24}$. Sin embargo, en otros estudios aparecen otras dimensiones que definen mejor a la disciplina: valorar a la persona holísticamente, teniendo en cuenta sus necesidades tanto físicas como psicológicas, prevenir la enfermedad y promocionar la salud ${ }^{17,20,24}$

En España, parece seguir existiendo una gran confusión con la identificación de los profesionales según su denominación ${ }^{23}$. Asimismo, los estudios realizados en el Reino Unido, EE.UU e Italia muestran cierta confusión entre el papel de las enfermeras cualificadas con la de los asistentes de sa$\operatorname{lud}^{17}$, las "Registered Nurses $»^{22}$ y otras figuras como las de auxiliar ${ }^{15}$.

\section{Independencia}

Se refiere a la independencia o autonomía que la sociedad cree que tienen las enfermeras para hacer juicios clínicos y tomar decisiones. Si se considera que tienen un campo de acción propio en el que pueden tener iniciativa, o por el contrario lo que hacen se deriva de una orden de los médicos o son consideradas sus ayudantes.

Mayoritariamente uno de los papeles asociados a las enfermeras por la población es la de ayudar al médico ${ }^{15,18,20,21,24}$, lo que apunta a que aún sigue vivo el viejo estereotipo de ayudante del médico. Parece que hay un escaso reconocimiento de la evolución de la profesión y del esfuerzo de las enfermeras por definir su propio espacio de autonomía ${ }^{15}$, no percibiéndose una actividad independiente a la del médico y con suficiente autonomía sobre el cuidado de los pacientes ${ }^{17,18,23-25}$.

\section{Formación}

Se abarca el nivel de formación que se les presupone a las enfermeras, si se consi- 
dera o no que la Enfermería es una carrera universitaria y el nivel de conocimientos que se cree que tienen.

Se puede apreciar una tendencia al cambio en la visión de la población española. Se pasa de un desconocimiento generalizado sobre la formación que reciben las enfermeras ${ }^{21}$ o de la concepción de que con vocación y el graduado es suficiente ${ }^{23}$, a la consideración no unánime por otro lado, de que la enfermería es una carrera universitaria ${ }^{18}$. Asimismo, dos de los estudios ${ }^{23,24}$, siendo ambos españoles y de la misma época, coinciden en que para la mayoría de usuarios la profesión ha mejorado sus conocimientos en los últimos años. Todo lo anterior contrasta con, otro estudio más reciente ${ }^{17}$ en el que el porcentaje de los encuestados que considera que están bien formadas es muy bajo.

Finalmente, no sólo parece haber cierta confusión en cuanto a la evolución de los estudios, sino que también para establecer grados académicos distintos en función del puesto de trabajo ${ }^{25}$, o de diferenciarla de otras figuras ${ }^{15,21}$.

\section{Atributos de las enfermeras}

Incluye los atributos, características, rasgos o cualidades más significativas que son asociados con la enfermería o cómo se describe a las enfermeras. Los aspectos que estaban más presentes en el conjunto de los artículos en orden decreciente se resumen en que sean profesionales ${ }^{16,17,19}$, amables $^{16,17,22}$, habilidosas ${ }^{15,19,22}$, pacientes $^{15,16}$, eficientes/eficaces ${ }^{16,22}$, expertas ${ }^{22}$, con conocimientos ${ }^{19}$ o inteligentes ${ }^{17}$. Destacando que estos atributos culturalmente están tipificados como femeninos ${ }^{26}$.

\section{Salario}

Aborda el salario que perciben las enfermeras y si la población estima que es el adecuado o no. Ninguno de los estudios que analizan dicho aspecto es español. Se ve una tendencia de la población encuestada a subestimar el potencial de ingresos de las enfermeras ${ }^{15,17,22}$.

\section{Confianza en el profesional de enfermería}

Se refiere a si la población confía en los profesionales enfermeros y en qué circunstancias.

A pesar de que la confianza que otorga la población a la labor de las enfermeras sea alta $^{24}$ y se confíe en ellas para manejar tareas clínicas rutinarias sin la supervisión de un médico ${ }^{22}$, esta confianza aún no es plena en tres particularidades. En primer lugar, con respecto a lo que dicen, ya que gran parte de la población prefiere constatar con el médico el consejo de salud que le haya podido dar la enfermera ${ }^{18,23}$. En segundo lugar, respecto a otros aspectos que no sean tareas asociadas desde antaño a la enfermería, como la cura de heridas o la toma de la tensión arterial ${ }^{25}$, y en tercer lugar, para el cuidado de enfermos a domicilio ${ }^{21}$.

\section{Género vinculado a la enfermería}

Esta categoría trata sobre si la población sigue pensando en la enfermería como una profesión de mujeres, o si por el contrario se va dando una normalización en la creencia de que es una profesión que puede ser ejercida igualmente por ambos sexos.

En el estudio más antiguo ${ }^{27}$ se afirma que la imagen pública de la enfermería continúa siendo firmemente estereotipada en base al género. No obstante, Renedo y col (1989) afirman que la mayoría considera que las enfermeras deben ser de ambos sexos, coincidiendo con estudios más recientes ${ }^{18,20}$. Asimismo, la percepción de la población sobre la enfermería como profesión estrictamente femenina está disminuyendo ${ }^{22}$, no importándole a la mayoría de los encuestados recibir cuidados de un hombre o de una mujer ${ }^{22}$ y habiendo decrecido el porcentaje de personas que cree que son mejores las mujeres ${ }^{17}$.

\section{DISCUSIÓN}

La imagen social de la enfermería constituye un tema amplio pudiendo ser analizada en diversos ámbitos. En esta revisión se ha tomado como foco de estudio a la población. Los resultados obtenidos deben ser 
considerados con cautela porque los estudios pertenecen a épocas y países diferentes. Henderson afirmó que las diferencias existentes entre los países en cuanto a las funciones que desempeñan las enfermeras y su preparación hacen insostenible un concepto universal de la enfermería ${ }^{28}$. Más aún, Domíngez-Alcón manifestó que «ninguna ocupación puede ser seguida de forma inteligente si no se ilumina con la luz de su historia" ${ }^{7}$.

No obstante, tras analizar las publicaciones, se han obtenido resultados que convergen hacia unas tendencias comunes. Parece que los resultados de los estudios muestran que la sociedad tiene una buena opinión sobre la enfermería, independientemente del país de estudio ${ }^{18,20-23,25}$. Cabe resaltar que la población tiene una actitud de respeto hacia la profesión ${ }^{18,20,21}$ y que como carrera universitaria es vista como una opción positiva. Asimismo, y no estando confrontado con lo anterior, en países tan dispares como Reino Unido, EE.UU., Cuba o España, parece existir la creencia de que las enfermeras no gozan del estatus que tienen los médicos a nivel profesional. Esto es lógico teniendo en cuenta los orígenes y la evolución de ambas profesiones.

Otro punto de aproximación es que el trabajo enfermero es descrito mediante muchas actividades, lo que puede explicar que se confíe en ellas sobre todo para tareas asociadas históricamente a la profesión. Cabe destacar que en algunos estudios se empiezan a mencionar funciones de prevención y promoción de la salud como áreas de enfermería ${ }^{17,20,24}$. Pero la confianza de la población en estas áreas no está consolidada ${ }^{18,23}$. Esto puede ser una consecuencia de que las enfermeras tiendan a ver en las habilidades técnicas una garantía de prestigio profesional ${ }^{2}$. Hemos de tener en cuenta que la visión que tiene el público de la enfermería varía en función de cómo se comporte ésta ${ }^{6}$. Por ello, se puede concluir que la población continúa desconociendo cuál es el quehacer diario y el foco de la enfermería, que es el cuidado de la persona, de la familia y de la comunidad dentro del marco de una relación interpersonal de transformación mutua. Por ello, los profesionales deberían plantearse cuál es la imagen que se está proyectando a la sociedad y cuál es la que se quiere forjar. "La práctica enfermera es mucho más que el cumplimiento de múltiples tareas. Requiere de una agudeza intelectual que, vista desde el exterior, parece indicar intuición, ya que se trata de acertar a escoger una acción pensada, reflexionada, que responda a las necesidades particulares de la persona ${ }^{2}$. Asimismo, que el campo de actuación de la enfermería no sea reconocido por el público, parece ser la consecuencia de que no se vea el trabajo y funciones que desempeñan las enfermeras. Tal y como concluyen Sridevy \& Prassanna Baby ${ }^{6}$, la falta de reconocimiento profesional del público en lo que se refiere a la capacidad enfermera, parece resultar de la invisibilidad de las funciones de la enfermería a la mayoría del público.

Los resultados parecen indicar que la enfermería no es una profesión tan estrechamente vinculada a la feminidad como cabía esperar. Progresivamente se va produciendo un cambio de la concepción que el público tiene de la profesión, considerándola como una opción más, de desarrollo profesional para los hombres.

En general, y siendo un aspecto común a varios países, parece existir cierto grado de confusión con respecto a las distintas figuras de enfermería, sus denominaciones y el rol que desempeñan. Esto puede deberse en primer lugar a la existencia de diferentes figuras y especialidades de enfermería, y en segundo, por los cambios que se han ido produciendo a lo largo de la historia tanto a nivel académico como profesional. El que durante estos últimos años se estén dando aún más cambios, puede hacer que esta cuestión quede sin resolver o que incluso se genere una mayor confusión.

Parece haber algunas tendencias en la sociedad que hacen que la enfermería sea vista bajo algunos prismas que no son un reflejo fiel al quehacer enfermero o que tienen una visión parcial de la misma, si se consideran las funciones del Código Deon- 
tológico de enfermería. Aunque sea algo común a todas las profesiones, hay que considerar que es posible que exista una disparidad en cuanto al auto-concepto que tienen las enfermeras, la percepción que tienen sobre cómo las ve la sociedad ${ }^{29,30}$, y la imagen social de la enfermería. El estudio realizado por Sridevy \& Prassanna Baby $^{6}$ reveló que la imagen pública de la enfermería no se correspondía con la imagen que las enfermeras tenían sobre ellas mismas.

Partiendo de la base de que "la enfermería como profesión constituye un servicio encaminado a satisfacer las necesidades de salud de las personas sanas o enfermas, individual o colectivamente $»^{31}$, el plantearse hasta qué punto la población lo percibe así puede ser oportuno y hasta necesario, ya que si desconocen lo que hacen las enfermeras, no recurrirán a ellas para beneficiarse de sus servicios.

Finalmente, cabe señalar que los estudios no están exentos de limitaciones derivadas principalmente del rigor y validez metodológica de los estudios hallados. El diseño de los estudios es cuantitativo y el instrumento de medida empleado son cuestionarios con preguntas principalmente cerradas o semicerradas, lo que puede condicionar las respuestas y constituir una fuente de sesgos que dificulten la obtención de una visión global del fenómeno explorado ${ }^{32}$. A ello se suma que algunos instrumentos carecen de validez, ya que la mayoría no describen en base a qué fueron desarrollados y no han sido pilotados previamente, y ninguno de ellos ha demostrado su fiabilidad. Asimismo, es razonable cuestionarse si las muestras de los estudios son representativas y si se pueden generalizar los resultados a la población tal y como lo hacen dos de los estudios ${ }^{18,25}$. Por ello, sería discutible la validez interna y externa de algunos estudios.

Puesto que estamos inmersos en un profundo cambio de los estudios universitarios (derivados del Plan de Bolonia) en el que se potencian los estudios de postgrado, y que sin duda, van a contribuir a la mejora de la imagen de la enfermería ${ }^{6}$, en un futuro podrían plantearse nuevas in- vestigaciones para evaluar el impacto del cambio de los estudios universitarios de enfermería sobre la opinión que la población tiene de la disciplina y de la profesión.

\section{CONSIDERACIONES FINALES}

Del análisis temático de las publicaciones han emergido ocho temas que pueden ayudar a explicar la visión que tiene la sociedad sobre la enfermería y la evolución que ha sufrido.

De esta revisión se puede concluir que la enfermería es una profesión poco visible y profundamente desconocida, ya que la sociedad todavía no reconoce plenamente la competencia, autonomía e independencia de las enfermeras, existiendo una tendencia a verla como una profesión inferior a la medicina y bajo su sombra. De modo que la mayoría de la población ignora que la enfermería tiene un campo competencial propio, relacionándola estrechamente con "tareas" y confiando en ella para las actividades que se le han asociado tradicionalmente. No obstante, se empiezan a vislumbrar otras funciones del personal de enfermería, y se va percibiendo como una profesión menos relacionada con la feminidad y más preparada que antaño.

El comportamiento propio de los profesionales tiene un efecto tanto sobre la imagen como sobre el conocimiento que el público tiene sobre la enfermería. Como profesión de servicio que es, esto es de suma importancia ya que si la población desconoce cuál es el quehacer de las enfermeras, no podrá recurrir a ellas. Asimismo, consideramos importante que los profesionales de enfermería reflexionemos sobre lo que hemos logrado y dónde estamos, para así poder definir bien lo que somos y lo que queremos ser y proyectar a la sociedad. Si como profesión y disciplina no somos capaces de "dar voz" a lo que somos y podemos ofrecer, difícilmente otros profesionales y la sociedad entenderán nuestra esencia.

Para concluir, a la hora de analizar una cuestión tan compleja como es la imagen que tiene la sociedad sobre la Enfermería sería conveniente tener en cuenta dos aspectos. Por un lado, considerar el país del 
estudio así como definir lo que representa una enfermera a nivel competencial, por las diferencias que se pueden dar. Por otro, realizar estudios que posibilitaran una mayor expresión de los participantes mediante preguntas abiertas, realizando estudios cualitativos o combinando metodologías, que respondan mejor a la pregunta de investigación y permiten profundizar más sobre el fenómeno en cuestión.

\section{Agradecimientos \\ Nos gustaría agradecer a la Cátedra Ma- ría Egea por el apoyo recibido.}

\section{BIBLIOGRAFÍA}

1. Cuxart i Ainaud, N. En equipo de investigación de profesiones y organizaciones de la Universidad de Barcelona. Enfermeras hoy: Cómo somos y cómo nos gustaría ser. Conclusiones del estudio sociológico 2004. Collegi Oficial d'Infermeria de Barcelona. Barcelona. 2005. Disponible en: http://212.9.72.18/uploadsBO/Generica/ Documents/ENFERMERAS\%20HOY\%20MAQUETAT.PDF.

2. Kèrouac S, Pepin J, Ducharme F, Duquette A, MaJOR F. El Pensamiento Enfermero. Barcelona: Masson, 1996.

3. Takase M, Kershaw E, Burt L. Does Public Image of Nurses Matter? J Prof Nurs 2002; 18: 196-205.

4. Cunningham A. Nursing stereotypes. Nurs Stand 1999; 13: 46-47.

5. Fernández C, Pérez M. Así nos muestran, ¿Así somos? Cultura de los Cuidados. 2005; Año IX, 18: 98-101.

6. SRidevy, Prassanna Baby. Public verses self image of nurses. Int J Nurs Educ 2010; 2: 5054.

7. Carrasco MC, Márquez M, Arenas J. Antropología-enfermería y perspectiva de género. Cultura de los Cuidados 2005; Año IX, 18: 52-59.

8. IruRtia, J. Comunicación de la Enfermería con la sociedad. V Congreso Nacional de Enfermería en Ostomías. 2004. [En línea: noviembre de 2004]. Disponible en: http:// www.estomaterapia.es/Congresos/Sede/ congreso2004/documentos/Ponencias/8_ Comunicacion\%20de\%20Enfermeria.pdf. [Acceso marzo de 2011]. Ponencia.
9. Hughes L. The public image of the nurse. Adv Nurs Sci 1980; 2: 55-72.

10. Kalisch B, Begeny S, Neumann S. The image of the nurse on the Internet. Nurs Outlook 2007; 55: 182-188.

11. Mejias-Padilla V. ¿Por qué intervenir en la elaboración de la política de enfermería? Enfermería Actual en Costa Rica 2007; 28: 4-5. Editorial.

12. Scottish Intercollegiate Guidelines Network. SIGN 50: A guideline developer's handbook. A guideline developer's handbook. Disponible en: http://www.sign.ac.uk/. [Acceso: marzo de 2011]. ISBN 9781905813254.

13. Dixon-Woods M, Agarwal S, Jones D, Young B, Sutton A. Synthesising qualitative and quantitative evidence: a review of possible methods. J Health Serv Res Policy 2005; 10: 45-53.

14. Domínguez Alcón C. Imagen de la enfermera en la sociedad. Rev Rol Enf 1985; 83: 66-72.

15. Prandi R, Ferri P. Enfermeros: ¿quienes somos? Un estudio transversal de la imagen social del enfermero en la ciudad de Módena (Italia). Ágora de enfermería 2007; 3: 11561159.

16. Casanovas I, Lázaro P, Mitjans J, Reixach M. Papel de la enfermera/o desde el punto de vista del usuario. Enferm Clin 1995; 5: 61-68.

17. Stuart P. What the public say. Nurs Stand 2004; 18: 14-15.

18. Remirez J, Pereda E, Delgado H, Delgado M. Enfermería y futuro: su evolución, ¿credibilidad? ENFURO 2010; 113: 15-18.

19. Donelan K, Buerhaus P, DesRoches C, Dittus R, Dutwin D. Public perceptions of nursing careers: the influence of the media and nursing shortages. Nurs Econ 2008; 26: 143-165.

20. Hernández LL, Rodriguez ME, JimÉnez M, Rangel F. Imagen social de la enfermería. Rev Cubana de Enferm 2003; 19: 250-269.

21. Renedo P, Canteras M, Santonja F, Cerezo M, HuERTAS J, IgNOTO M. Imagen social de la Enfermería. Encuesta al usuario. Rev Rol Enf 1989; 12: 31-37.

22. BEgany T. Your image is brighter than ever. RN 1994; 57: 28-35.

23. MaRTínez JR. Enfermería: opina la sociedad. Enfermería Científica 1996; 170-171: 4-8.

24. Vázquez M, Casals J, Aguilar P, Ramírez M, GóMEZ M, VILLaRín V. Percepción por el usuario de la imagen de enfermería. Enferm Clin 1997; 7: 105-111. 
25. Pedre M, Pita M, Valiño C. Imagen social de la enfermería: un vistazo al espejo público. Asociación Española de Enfermería Urológica 2004; 89: 32-33.

26. Kaler SR, Levy DA, Schall M. Stereotypes of professional roles. Image J Nurs Sch 1989; 21: 85-89.

27. Scott SD, Lower MS. Image of Nursing: Perceptions of nurses, physicians and the public. The Missouri Nurse 1988; 57: 18-19.

28. Henderson V. The concept of nursing. J Adv Nurs 2006; 53: 21-34. Publicado por primera vez en J Adv Nurs 1978; 3: 113-130.

29. Takase M, Maude $P$, Manias E. Impact of the perceived public image of nursing on nurses' work behaviour. J Adv Nurs 2006; 53: 333-343.
30. TAKase M, Kershaw E, Burt L. Nurse-environment misfit and nursing practice. J Adv Nurs 2001; 35: 819-828.

31. Consejo G de Colegios de Diplomados en Enfermería. Código deontológico de la enfermería española. Resolución n⿳ำ 32/89, del Consejo General de Enfermería, por la que se aprueban las normas deontológicas que ordenan el ejercicio de la profesión de Enfermería de España con carácter obligatorio. 1989. Sociedad Española de Enfermería Intensiva y Unidades Coronarias. Disponible en http://www.seeiuc.com/profesio/codigo. htm\#Preambulo.

32. Murphy-Black T. Los cuestionarios. En: Gerrish K, Lacey A. Investigación en enfermería. Madrid: McGraw-Hill, 2008, p. 367-382. 